04,11

\title{
Структурные механизмы и термодинамическое описание изосимметрийного орбитального упорядочения в редкоземельных манганитах
}

\author{
(C) М.П. Ивлиев, В.П. Сахненко
}

Научно-исследовательский институт физики при Южном фредеральном университете, Ростов-на-Дону, Россия

E-mail: ivlievmp@rambler.ru

Поступила в Редакцию 4 июня 2019 г.

В окончательной редакции 4 июня 2019 г.

Принята к публикации 4 июня 2019 г.

Показано, что наблюдаемые особенности орбитального фазового перехода в перовскитах $\mathrm{AMnO}_{3}$ $(\mathrm{A}=\mathrm{La}, \operatorname{Pr}, \ldots, \mathrm{Lu})$ обусловлены возникновением при высоких температурах ротационных искажений кубической решетки, вызывающих смещения анионов, которые создают „внутреннее поле“, по симметрии совпадающее с параметром порядка, описывающим упорядочение октаэдров, искаженных вследствие эффекта Яна-Теллера (орбитальное упорядочение). Влияние этого „поля“ существенным образом определяет тип орбитального упорядочения, его температуру и род фазового перехода. Сравнительный анализ орбитально упорядоченных состояний выполнен для всего ряда $\mathrm{AMnO}_{3}$, как в рамках феноменологической теории фазовых переходов, так и на основе предложенной в статье трехминимумной многоподрешеточной модели. Обсуждается, обусловленная особенностями структуры, специфика магнитных переходов в манганитах.

Ключевые слова: манганиты, ромбические фазы, фазовый переход порядок-беспорядок, орбитальное и ротационное взаимодействие, упругие и магнитные свойства.

DOI: 10.21883/FTT.2019.11.48408.505

\section{1. Введение}

Манганиты семейства лантана со структурой перовскита обладают целым рядом уникальных физических свойств, многие из которых связанны с наличием в области низких температур разнообразных фазовых переходов - магнитных, зарядовых и т.п. Поэтому большинство работ посвящено исследованию свойств манганитов именно при низких температурах [1-5]. Однако для понимания и правильной интерпретации того, что там наблюдается необходимо учитывать структурные особенности фазы, в которой происходят такие низкотемпературные фазовые переходы.

В этой области кристаллы имеют ромбическую симметрию $\left(D_{2 h}^{16}-\right.$ Pnma, Pbnm $)$, которая возникает после ряда высокотемпературных фазовых переходов [6,7]. Характерной особенностью ромбической решетки является то, что она формируется за счет смещений анионов, которые в первом приближении можно трактовать, как повороты октаэдров $\mathrm{MnO}_{6}$ относительно осей симметрии исходной кубической решетки (ротационные искажения структуры, tilting). Аналогичной структурой обладают многие другие перовскиты, в частности, ортоферриты [8]. Однако отличие манганитов в том, что у них наряду с ротационным упорядочением возникает и орбитальное, проявляющееся ориентационным упорядочением октаэдров $\mathrm{MnO}_{6}$, тетрагонально деформированных вследствие эффекта Яна-Теллера $[5,7,9]$. Сопоставление свойств кристаллов, имеющих структуру перовскита, в которых наблюдаются по-отдельности аналогичные упорядочения либо орбитальные, либо ротационные, дает основание полагать, что структурная основа, на которой в дальнейшем реализуются уникальные физические свойства манганитов, формируется именно за счет сочетания ротационных и орбитального упорядочений.

В работе основное внимание уделено исследованию изосимметрийного фазового перехода (ФП) между ромбическими фазами $\mathrm{O}$ и $\mathrm{O}^{\prime}$, где $\mathrm{O}-$ ротационно упорядоченная фаза симметрии $\mathrm{Pbnm}\left(\mathrm{D}_{2 h}^{16}, \mathrm{Z}=4\right)$, а $\mathrm{O}^{\prime}-$ фаза, в которой наряду с ротационным упорядочением появляется и орбитальное [6,7,10-13]. Структура фазы О является слабо искаженной модификацией кубической структуры. Структура фазы $\mathrm{O}^{\prime}$ также близка к кубической [14]. Однако при орбитальном фазовом переходе $\mathrm{O} \rightarrow \mathrm{O}^{\prime}$ деформации решетки относительно фазы О и одноосные, и ромбические, характеризуемые соответственно отношениями $(2 b a)^{1 / 2} / c, b / a(c, b, a-$ постоянные решетки ромбической фазы), существенно увеличиваются. В результате соотношения между основными параметрами решетки меняются, что приводит к изменению основного структурного мотива, но при этом фазы О и $\mathrm{O}^{\prime}$ остаются изосимметричными $[7,9,15]$. Также при переходе наблюдаются аномалии упругих свойств [16,17]. Кроме того, в $\mathrm{LaMnO}_{3}$ упомянутые изменения происходят „скачком“ ${ }^{6}[6,15]$, а в других $\left(\mathrm{PrMnO}_{3}, \mathrm{NdMnO}_{3}\right)$ непрерывным образом [10,11].

Обычно орбитальный фазовый переход трактуется как появление ориентационного дальнего порядка в подси- 
стеме небольших локально упорядоченных блоков, состоящих из одного или нескольких деформированных октаэдров [18]. При этом считается, что в области $T \gtrsim T_{J T}$ $\left(T_{J T}\right.$ - температура орбитального ФП) в подсистеме октаэдров орбитальное упорядочение либо полностью отсутствует, либо проявляется как „остаточное“ явление. Причины, по которым ФП становится непрерывным, до настоящего момента так и не были выяснены. Попытка трактовать его, как некий „размытый“ переход с широкой областью сосуществования фаз $\mathrm{O}$ и $\mathrm{O}^{\prime}$, вступает в противоречие с тем, что он сопровождается резко выраженными температурными аномалиями решеточных постоянных $[10,11]$. Также возникает вопрос о роли ротационных упорядочений в реализации орбитального ФП. В ряде относительно простых случаев ротационные и орбитальные упорядочения рассматривались совместно на основе феноменологической теории [19]. Однако в большинстве теоретических работ, в которых исследуются орбитальные ФП в манганитах, наличие ротационных искажений структуры вообще не учитывается.

В работе в рамках феноменологической теории и на основе статистической модели с учетом наличия ротационных упорядочений исследуются условия возникновения орбитального упорядочения (т.е. его тип, род ФП и т.п.) для всего ряда редкоземельных манганитов. Анализируются факторы, ответственные за возникновение в фазе $\mathrm{O}^{\prime}$ значительных одноосных и ромбических деформаций. Выясняются причины, по которым при ФП, существенно меняющим основной структурный мотив кристалла, его симметрия не меняется. Устанавливаются особенности структуры, которые способствуют появлению магнитной сверхструктуры в манганитах, сопровождаемой возникновением несоразмерных фаз и магнитоэлектрических состояний [4].

\section{2. Исследование орбитального упорядочения в манганитах}

Электронная конфигурация $\mathrm{Mn}^{3+}$ в анионном октаэдре $-d^{4}\left(t_{2 g}^{3} e_{g}^{1}\right)$, при этом уровень $e_{g}$ вырожден. Снятие вырождения осуществляется посредством деформации октаэдра (эффект Яна-Теллера). При растяжении октаэдра вдоль одной из осей $C_{4}$, например, вдоль оси $z$, энергия орбитали $d_{Z^{2}}\left(d_{2 z^{2}-x^{2}-y^{2}}\right)$ уменьшается, а энергия орбитали $d_{x^{2}-y^{2}}$ увеличивается, что приводит к локализации электрона на орбитали $d_{Z^{2}}$, т.е. к ее заполнению [20].

Катион $\mathrm{Mn}^{3+}$ в октаэдрическом окружении относится к числу „сильно деформирующих“ ЯТ катионов [21]. Согласно $[9,18,22,23]$ устойчивые искажения октаэдров в манганитах наблюдаются при температурах, заметно превышающих температуры орбитального ФП. Это позволяет орбитальный ФП трактовать как упорядочение в системе тетрагонально искаженных (растянутых) октаэдров, имеющих в симметричной фазе три равноправные ориентации вдоль направлений типа [001]

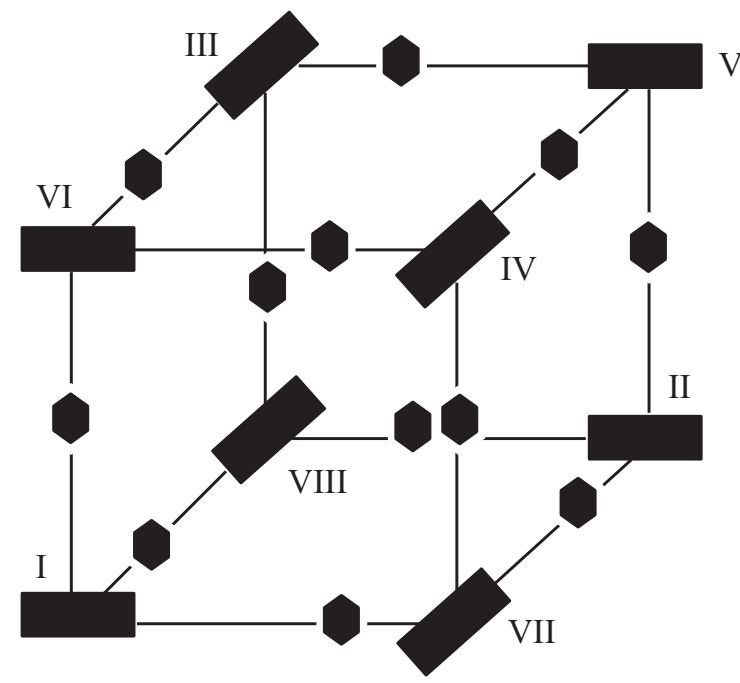

Рис. 1. Орбитальное упорядочение и расположение анионов, характеризуемое ПП $\left(0,0, \chi_{3}\right)$. Римскими цифрами обозначены номера подрешеток катионов $\mathrm{Mn}^{3+}$.

кубической решетки. При этом упорядочение рассматривается как переход „порядок-беспорядок“, обусловленный перераспределением вероятности ориентаций по трем кристаллографически эквивалентным позициям (КЭП) $[20,24,25]$.

В рассматриваемых манганитах возникает орбитальное упорядочение, характеризуемое ПП $\left(0,0, \chi_{3}\right)[7,24]$. Трехкомпонентный ПП $\chi$ преобразуется по неприводимому представлению (НП) $\mathrm{M}_{2}^{+}\left(\mathbf{k}=1 / 2\left(\mathbf{b}_{1}+\mathbf{b}_{2}\right)\right)$ $\left(\mathbf{b}_{i}\right.$ - векторы обратной решетки) [26] и описывает упорядочения антиферродисторсионного типа. В отсутствие ротационных искажений симметрия упорядоченного фазового состояния (ФС) $P 4 / \mathrm{mbm}, Z=2\left(D_{4 h}^{5}\right)$. Оно изображено на рис. 1.

Описание упорядочений, характеризуемых ПП $\chi$ осуществляется на основе трехминимумной $(3 \mathrm{~min})$ восьмиподрешеточной модели [24,27]. Типичная диаграмма фазовых состояний с критическими ПП $\chi$ и $\eta$ имеет вид (рис. 2).

Фаза I с симметрией $P 4 / \mathrm{mmm}(\mathbf{k}=0)\left(D_{4 h}^{1}\right)$ описывается ПП $\left(\eta_{1}, 0,0,0,0\right)$ где $\chi_{1}>0$. Фаза II симметрии $P 4 / m b m, Z=2$, характеризуется ПП $\left(\eta_{1}, 0,0,0, \chi_{3}\right)$, $\eta_{1}<0$, а фаза III симметрии $\operatorname{Im} 3(Z=8)\left(T_{h}^{5}\right)$, описывается ПП $(0,0, \chi, \chi, \chi)$. В дальнейшем нас будет интересовать область $A<B<0$ диаграммы (рис. 2).

Для описания упорядочений типа $\left(00 \chi_{3}\right)$ введем функции $n_{i L}(i=1,2,3 ; L=\mathrm{I}, \ldots, \mathrm{VIII})$, характеризующие вероятности ориентации заполненной орбитали типа $d_{Z^{2}}$ ЯТ катиона в подрешетке $L$ вдоль направления $i$, другими словами - вероятности ориентации по $i$-й КЭП в подрешетке $L$. Нумерация подрешеток дана римскими цифрами на рис. 1.

Упорядочение, изображенное на рис. 1, характеризуется критическим ПП $\chi_{3}$ и сопутствующим ПП $\eta_{1}$ 


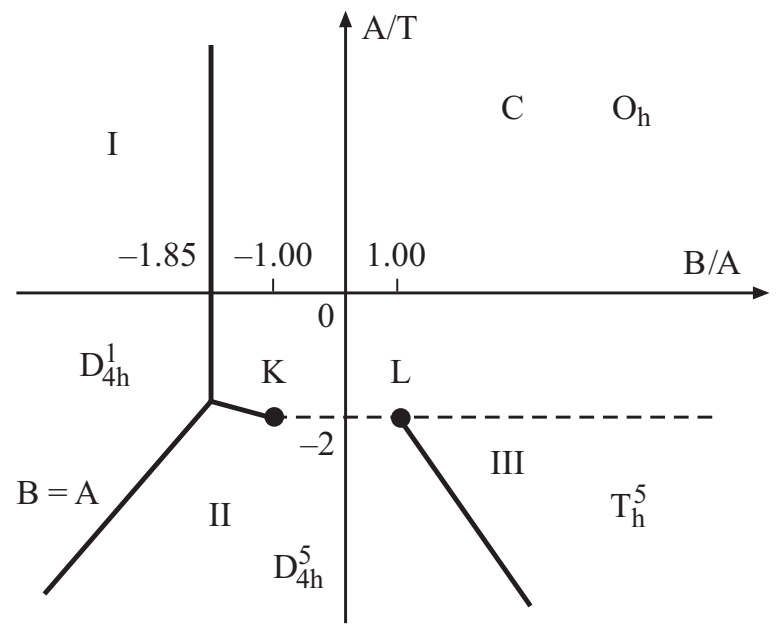

Рис. 2. Диаграмма фазовых состояний 3 min восьмиподрешеточной модели с критическими ПП $\chi$ и $\eta$.

$\left(\eta_{1} \sim \chi_{3}^{2}\right)$ и описывается следующим набором функций $n_{i L}\left(0 \leq n_{i L} \leq 1\right)$

$$
\begin{gathered}
n_{i \mathrm{I}}=n_{i \mathrm{II}}=n_{i \mathrm{~V}}=n_{i \mathrm{VI}} \quad(i=1,2,3), \\
n_{j \mathrm{III}}=n_{j \mathrm{IV}}=n_{j \mathrm{VII}}=n_{j \mathrm{VIII}}(j=1,2,3), \\
n_{3 \mathrm{I}}=\frac{1}{3}\left(1+2 \eta_{1}\right), n_{2 \mathrm{I}}=\frac{1}{3}\left(1-\eta_{1}+\chi_{3}\right), \\
n_{1 \mathrm{I}}=\frac{1}{3}\left(1-\eta_{1}-\chi_{3}\right), \\
\left.n_{3 \mathrm{III}}=\frac{1}{3}\left(1+2 \eta_{1}\right), n_{2 \mathrm{III}}=\frac{1}{3}\left(1-\eta_{1}-\chi_{3}\right)\right), \\
n_{1 \mathrm{III}}=\frac{1}{3}\left(1-\eta_{1}+\chi_{3}\right),
\end{gathered}
$$

$\eta_{1}$ - компонента двухкомпонентного

ПП

$\left(\eta_{1} \in \Gamma_{3}^{+}(\mathbf{k}=0)\right)$. По трансформационным свойствам компонента ПП $\eta_{1}$ аналогична компоненте тензора одноосных деформаций $U_{1}=2 u_{z z}-u_{y y}-u_{x x}$, поэтому основной вклад в энергию их взаимодействия описывается билинейным членом. При таком упорядочении образуются две подрешетки и возникает орбитальный порядок типа $C$ [28].

В отсутствие ротационных упорядочений конденсация ПП $\chi_{3}$ описывается термодинамическим потенциалом (ТП), который имеет вид

$$
F\left(\chi_{3}, \eta_{1}\right)=A \chi_{3}^{2}+3 B \eta_{1}^{2}+T \sum_{i=1}^{3} n_{i \mathrm{I}} \ln n_{i \mathrm{I}} .
$$

Константы $A$ и $B$ выступают в качестве феноменологических параметров теории.

Упорядочения, описываемые ТП, который по виду совпадает с (1), детально исследованы в [25]. В области $A<B<0.5 \mathrm{~A}, A<0$, термодинамический потенциал $F\left(\chi_{3}, \eta_{1}\right)$ описывает ФП первого рода из кубической в тетрагональную фазу $\left(\eta_{1}<0,0,0,0, \chi_{3}\right)$ с симметрией $P 4 / \mathrm{mbm}(Z=2)$ [24]. Условие $\eta_{1}<0\left(\eta_{1}>0\right)$ означает, что решетка сжата (растянута) вдоль тетрагональной оси [25]. Необходимо особо отметить, что согласно модели (1) условием реализации перехода первого рода является $B<0.5 A<0$. Это означает, что при таком переходе будет наблюдаться „размягчение“ не только по ПП $\chi$, но и по ПП $\eta$, а вследствие билинейной связи ПП $\eta$ с деформациями $U$, то и относительно одноосных деформаций $U$. Соответственно будет наблюдаться и „размягчение“ модуля $c_{11}-c_{12}$.

\section{3. Влияние ротационных упорядочений на формирование орбитального порядка в манганитах}

Ротационные упорядочения в фазе О характеризуются набором параметров порядка $\varphi \varphi \psi$ (в обозначениях Глейзера [12] $\left.-\left(a^{-} a^{-} c^{+}\right)\right)$. Параметр порядка $\varphi$ преобразуется по НП $R_{4}^{+}\left(\mathbf{k}=1 / 2\left(\mathbf{b}_{1}+\mathbf{b}_{2}+\mathbf{b}_{3}\right)\right)$, а ПП $\psi$ - по $\mathbf{M}_{3}^{+}\left(\mathbf{k}=1 / 2\left(\mathbf{b}_{1}+\mathbf{b}_{2}\right)\right)[13,26]$. Параметры $\varphi_{i}$ и $\psi_{i}$ описывают антифазные и синфазные „повороты“ октаэдров вокруг оси $i(i=x, y, z)$ соответственно.

Главный член, характеризующий взаимодействие орбитального и ротационных ПП имеет следующий вид [24] (возможность существования такого инварианта косвенным образом следовала из работ $[7,19])$

$$
H_{\text {int }}(\psi, \varphi, \chi)=\delta\left(\chi_{3} \psi_{3} \varphi_{1} \varphi_{2}+\chi_{2} \psi_{2} \varphi_{3} \varphi_{1}+\chi_{1} \psi_{1} \varphi_{2} \varphi_{3}\right)
$$

Из $H_{\text {int }}(\psi, \varphi, \chi)$ следует, что в случае $\varphi_{1} \neq 0, \varphi_{2} \neq 0$, $\psi_{3} \neq 0$ параметр порядка $\chi_{3}$, описывающий орбитальное упорядочение, также будет отличен от нуля. Соответственно конденсат параметров порядка, характеризующий фазу $\mathrm{O}$ в лантаноидных манганитах, будет $\varphi \varphi \psi_{3} \chi_{3}$.

Наличие члена $\varphi_{i} \varphi_{j} \psi_{k} \chi_{k} \quad(i \neq j \neq k)$ означает, что совокупность ротационных искажений типа $\varphi_{i} \varphi_{j} \psi_{k}$ кубической решетки, вызванных смещениями анионов, можно рассматривать как поле, термодинамически сопряженное ПП $\chi_{3}$. Поэтому орбитальный ФП из фазы О в фазу $\mathrm{O}^{\prime}$ фактически оказывается переходом во „внешнем поле“, которое определяет конденсирующуюся компоненту ПП $\chi$. Конденсат ПП в фазе $\mathrm{O}^{\prime}$ такой же как в фазе $\mathrm{O}-\left(\varphi \varphi \psi_{3} \chi_{3}\right)$, поэтому обе фазы имеют одинаковую симметрию, т.е. изосимметричны. Разница в том, что в фазе О параметр $\chi$ индуцирован „внешним полем“ и и проявляется слабо, а в фазе $\mathrm{O}^{\prime}$ он „Появляется“ вследствие потери устойчивости (т.е. как „собственный“) и существенно отличается по величине. Основное отличие фазы О от фазы $\mathrm{O}^{\prime}$ в том, что в первой основной структурный мотив формируется ротационным упорядочением, а во второй - орбитальным (рис. 1) [7,24].

Решетки ромбических фаз О манганитов очень близки к кубическим (особенно для $\mathrm{LaMnO}_{3}$ ) [6,14]. Наличием небольших деформаций структуры, обусловленных 


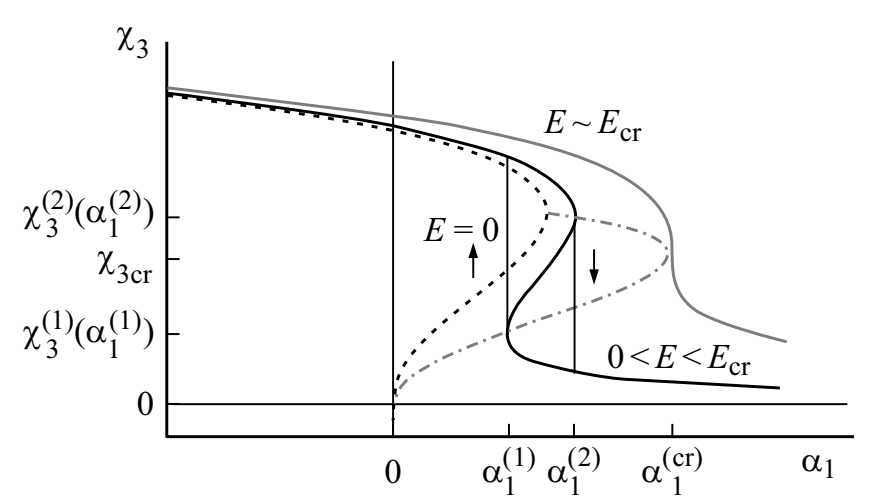

Рис. 3. Зависимости $\chi_{3}\left(\alpha_{1}\right)$, определяемые уравнением (2), при различных „внешних полях“, штриховой - при $E=0$, толстой и тонкой сплошными линиями при $0<E<E_{\text {cr }}$ и $E=E_{\text {cr }}$, соответственно, штрих-пунктирной обозначено геометрическое место точек, в которых $\left[\partial \chi_{3}\left(\alpha_{1}, E\right) / \partial \alpha_{1}\right]^{-1}=0$. Стрелками указано направление изменения ПП, $\uparrow-$ при охлаждении, $\downarrow-$ при нагреве.

ротационными ПП, которые не существенны для дальнейшего рассмотрения, можно пренебречь. Поэтому в качестве модельной системы рассматриваем кристалл, имеющий простую кубическую решетку, в узлах которой расположены ЯТ катионы, имеющие три равноправные ориентации, а наличие небольших ротационных искажений учитываем введением „внешнего поля“, которое действует на орбитальный ПП, по которому система теряет устойчивость.

На первом этапе характерные особенности орбитального ФП во „внешнем поле“ исследуем на основе феноменологической теории. Термодинамический потенциал для описания изосимметрийного ФП первого рода $O-O^{\prime}$ можно представить в виде

$$
\Phi_{J T}\left(\chi_{3}, E\right)=\alpha_{1} \chi_{3}^{2}+\alpha_{2} \chi_{3}^{4}+\alpha_{3} \chi_{3}^{6}+\ldots-E \chi_{3},
$$

где $E=\delta \varphi^{2} \psi, \alpha_{1} \rightarrow 0$ (так как ПП $\chi_{3}$ критический), $\alpha_{2}<0$ (так как переход І-го рода), $\alpha_{3}>0$. При этом уравнение состояния $\partial \Phi_{J T}\left(\chi_{3}, E\right) / \partial \chi_{3}=0$, определяющее зависимость $\chi_{3}$ от $\alpha_{1}$, а фактически $\chi_{3}$ от температуры $T$, имеет вид

$$
2 \alpha_{1} \chi_{3}+4 \alpha_{2} \chi_{3}^{3}+6 \alpha_{3} \chi_{3}^{5}-E=0
$$

Варианты зависимости $\chi_{3}\left(\alpha_{1}\right) \sim \chi_{3}(T)$ при различных „внешних полях“ приведены на рис. 3. Значения $E_{\mathrm{cr}}, \alpha_{1}^{(\mathrm{cr})}$ и $\chi_{3 \mathrm{cr}}$ определяются уравнениями (2) и $\partial^{2} \Phi_{J T}\left(\chi_{3}, E\right) / \partial \chi_{3}^{2}=0$

$$
E_{\mathrm{cr}}=\frac{16}{25} \frac{\alpha_{2}^{2}}{\alpha_{3}} \sqrt{\frac{-\alpha_{2}}{\alpha_{3}}}, \quad \alpha_{1}^{(\mathrm{cr})}=\frac{3}{5} \frac{\alpha_{2}^{2}}{\alpha_{3}}, \quad \chi_{3 \mathrm{cr}}^{2}=-\frac{1}{5} \frac{\alpha_{2}}{\alpha_{3}} .
$$

Также из рис. 3 следует, что в области $\chi_{3}>0$ при $0<E<E_{\text {cr }}$ зависимость $\chi_{3}\left(\alpha_{1}\right)$ имеет две устойчивые ветви: высокотемпературную - $\chi_{3}^{(1)}\left(\alpha_{1}, E\right)$ $\left(\chi_{3}^{(1)}\left(\alpha_{1}, E\right) \leq \chi_{3}^{(1)}\left(\alpha_{1}^{(1)}, E\right)\right)$ и низкотемпературную - $\chi_{3}^{(2)}\left(\alpha_{1}, E\right) \quad\left(\chi_{3}^{(2)}\left(\alpha_{1}, E\right) \geq \chi_{3}^{(2)}\left(\alpha_{1}^{(2)}, E\right)\right) . \quad$ Границы устойчивости этих ветвей определяются соотношениями $\left[\partial \chi_{3}^{(j)}\left(\alpha_{1}, E\right) / \partial \alpha_{1}\right]^{-1}=0(j=1,2)$. При фиксированном $E$ значения $\alpha_{1}(j) \sim T_{j}$ и $\chi_{3}^{(j)}\left(\alpha_{1}^{(j)}, E\right)$, определенные упомянутыми соотношениями, являются предельными для каждой из ветвей. Соответственно $\alpha_{1}^{(2)}-\alpha_{1}^{(1)} \sim T_{2}-T_{1}$ определяет величину температурного гистерезиса в цикле „нагрев-охлаждение“, а $\chi_{3}^{(1)}\left(\alpha_{1}^{(2)}, E\right)-\chi_{3}^{(2)}\left(\alpha_{1}^{(2)}, E\right)$ и $\chi_{3}^{(2)}\left(\alpha_{1}^{(1)}, E\right)-\chi_{3}^{(1)}\left(\alpha_{1}^{(1)}, E\right)$ - „скачок“ ПП $\chi_{3}$ при нагреве и охлаждении (рис. 3), т.е. фактически параметры петли гистерезиса.

Существенная особенность орбитального изосимметрийного ФП в рассматриваемых кристаллах заключается в том, что с ростом ротационных ПП величина поля $E$ растет, а когда $E$ достигает, либо превышает значение $E_{\mathrm{cr}}$, то этот переход трансформируется в непрерывный ФП. О том, что происходит такой „переход“ можно судить только по нелинейному росту ПП $\chi$ и связанных с ним физических характеристик, в частности параметров решетки.

Уравнение (2) подобно соответствующему уравнению для описания сегнетоэлектриков, испытывающих ФП первого рода во внешнем электрическом поле. Отличие в том, что в рассматриваемом случае величина $E$ не является постоянной, так как $E \sim \varphi^{2} \psi$, а ПП $\varphi$ и $\psi_{3}$ меняются при изменении ПП $\chi_{3}$. Однако, поскольку температура орбитального ФП заметно ниже температур, при которых появляются ПП $\varphi$ и $\psi_{3}$, то при $T \sim T_{J T}$ параметры $\varphi$ и $\psi_{3}$ оказываются в области насыщения. Соответственно их изменения, обусловленные „скачком“ ПП $\chi_{3}$ будут относительно малы (см., например, [15]). Поэтому при исследовании особенностей орбитального ФП в окрестности перехода изменениями ПП $\varphi$ и $\psi_{3}$ в рамках такого приближенного рассмотрения можно пренебречь. Более того, можно утверждать, что используемое приближение „постоянного поля“ дает возможность исследовать и дать адекватное описание основных характерных особенностей орбитального ФП при наличии ротационных искажений структуры в широком интервале температур.

Далее исследование орбитального ФП проведем на основе рассмотренной ранее трехминимумной модели (см. ч. 2). В этом случае конденсация ПП $\chi_{3}$ описывается ТП, в котором наряду с термодинамическим потенциалом (1), характеризующим вклад от орбитальных степеней свободы, учитывается взаимодействие ПП $\chi_{3}$ $\mathrm{c}$, „внешним полем“. Этот ТП имеет следующий вид

$$
F\left(\chi_{3}, \eta_{1}, E\right)=-E \chi_{3}+F\left(\chi_{3}, \eta_{1}\right),
$$

где $E=\delta \varphi^{2} \psi_{3}-$ внешнее поле, созданное ротационными ПП, действующее на ПП $\chi_{3}$. Равновесные значения параметров порядка $\chi_{3}, \eta_{1}$ определяются из системы уравнений состояния $\partial F\left(\chi_{3}, \eta_{1}, E\right) / \partial \chi_{3}=0$, $\partial F\left(\chi_{3}, \eta_{1}, E\right) / \partial \eta_{1}=0$.

Подбором значений модельных параметров $E$ и $B$ получено относительно реалистичное описание ФП в 

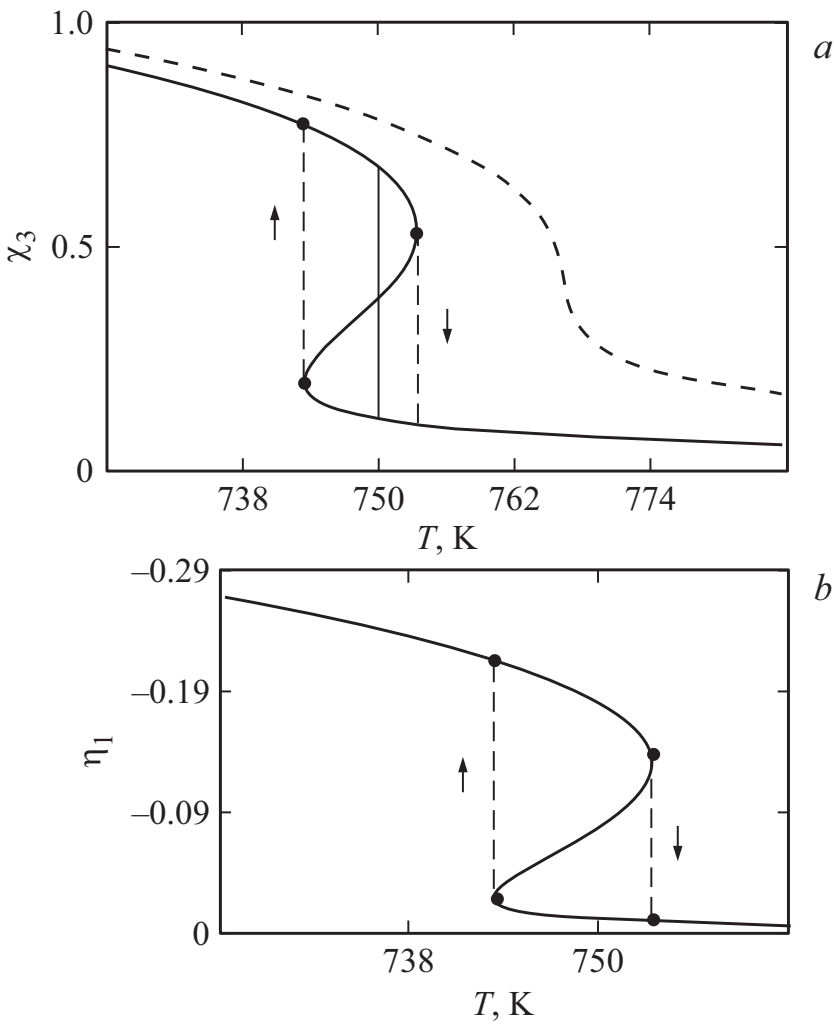

Рис. 4. $a-$ зависимость ПП $\chi_{3}(T)$, описываемая ТП (3), при $E=-0.01 A$ - сплошная линия, при $E=-0.03 A-$ штриховая линия, стрелками указано направление изменения ПП, $\uparrow-$ при охлаждении, $\downarrow-$ при нагреве; $b-$ зависимость ПП $\eta_{1}(T)$, определяемая ТП (3) при $E=-0.01 A$.

$\mathrm{LaMnO}_{3}$ [24]. При $E=-0.01 A, B=0.82 A$ находим, что при $T_{\mathrm{tr}}=-A / 0.317$ термодинамический потенциал фаз $1(O)$ и $2\left(O^{\prime}\right)$, характеризуемых $\chi_{31}=0.11$, $\eta_{11}=-8.9 \cdot 10^{-3} \quad$ и $\chi_{32}=0.665, \eta_{12}=-0.18$ равны. Между ними происходит ФП первого рода, сопровождаемый „скачком“ П П $\chi$ и $\eta$. Поскольку $T_{\mathrm{tr}} \sim 750 \mathrm{~K}$, то $A \sim-238 \mathrm{~K}$. Зависимости $\chi_{3}(T)$ и $\eta_{1}(T)$, описываемые ТП (3) при различных значениях внешнего поля имеют вид, изображенный на рис. $4, a, b$.

Низкотемпературная ветвь $\chi_{3}(T)$ устойчива в области $T \lesssim 753 \mathrm{~K}$, а высокотемпературная ветвь при $T \gtrsim 743 \mathrm{~K}$. Соответственно при охлаждении, при $T \sim 743 \mathrm{~K}$ будет наблюдаться „скачок“ ПП $\chi_{3}$ от $\sim 0.2$ $\left(\eta_{1} \sim-0.026\right)$ до $\sim 0.76\left(\eta_{1} \sim-0.217\right)$, а при нагреве, при $T \sim 753 \mathrm{~K}$ ПП меняется от $\sim 0.53\left(\eta_{1} \sim-0.12\right)$ до $\sim 0.1\left(\eta_{1} \sim-0.01\right)$, а температурный гистерезис будет $\sim 10 \mathrm{~K}$. Обращает на себя внимание то, что при переходе величина ПП $\eta_{1}$ меняется почти на порядок (рис. 4,b).

При подборе модельных параметров учитывались данные работ [22,23]. В этих работах из анализа и интерпретации структурных и спектроскопических данных следовало, что при ФП переходе первого рода в $\mathrm{LaMnO}_{3}$ $(T \sim 750 \mathrm{~K})$ параметр дальнего порядка, аналогичный
ПП $\chi_{3}$ испытывает „скачок“ от $\sim 0.1$ до $\sim 0.55-0.65$. Учет этих данных в совокупности с рядом других факторов (см. далее) позволил выбрать относительно реалистичные соотношения между основными параметрами модели.

\section{4. Обсуждение результатов}

В отсутствие „внешнего поля“ температура потери устойчивости симметричной фазы по ПП $\chi_{3}$ равна $T_{c} \sim 710 \mathrm{~K}$. Поскольку $B=0.82 A<0$, то при понижении температуры в случае $E=0, \chi_{3}=0$ система могла бы потерять устойчивость по ПП $\eta$ при $\sim 590 \mathrm{~K}$. Соответственно, модуль $c_{11}-c_{12}$ также должен был бы обратиться в нуль при $T \gtrsim 590 \mathrm{~K}$. Вследствие наличия такого „размягчения“, в окрестности изосимметрийного ФП $\mathrm{O} \rightarrow \mathrm{O}^{\prime}$ должны наблюдаться аномалии упругих свойств, а сам ФП происходит между фазами, „мягкими“ относительно одноосных деформаций. „Размягчение“ упругого модуля, характеризующего одноосные деформации, действительно наблюдалось в окрестности изосимметрийного ФП в $\mathrm{LaMnO}_{3}$ [17] и $\mathrm{La}_{0.88} \mathrm{Sr}_{0.12} \mathrm{MnO}_{3}$ [16]. Этот факт является прямым подтверждением того, что в рассматриваемом случае в соответствии с модельным описанием фазовый переход первого рода возникает вследствие взаимодействия ПП $\chi_{3}$ c „мягкой“ подсистемой, характеризуемой ПП $\eta$. При других механизмах формирования ФП первого рода, например, за счет сильного стрикционного взаимодействия, либо за счет сильного ангармонизма [29], упругий модуль в окрестности ФП размягчается в значительно меньшей степени.

„Мягкость“ фаз проявляется в том, что одноосные деформации в фазе $\mathrm{O}^{\prime}$ выражены на порядок сильнее чем в фазе $\mathrm{O}[14]$. Поскольку ПП $\eta_{1}<0$, то ромбическая решетка фазы $\mathrm{O}^{\prime}$ сжата по $z$ и растянута по $x, y$. При этом надо иметь в виду, что одноосные деформации решетки прямо пропорциональны ПП $\eta_{1}$, причем зависимость $U_{1}$ от $\eta_{1}$ близка к линейной [25]. Из-за „мягкости“ системы при ФП параметр $\eta_{1}$ увеличивается на порядок (рис. $4, b)$, что сопровождается резким ростом одноосных деформации в фазе $\mathrm{O}^{\prime}$. Таким образом вследствие орбитального упорядочения, описываемого ПП $\chi_{3}$, решетка кристалла станет тетрагональной, хотя и относительно близкой к кубической (рис. 1) с наличием небольших ротационных искажений. Их влияние проявится в том, что число подрешеток возрастет до четыpex, а симметрия кристалла понизится до ромбической.

Существование деформационной „мягкости“ (т.е. высокой восприимчивости) относительно одноосных деформаций во-многом обусловлено наличием в симметричной фазе октаэдров, тетрагонально деформированных вследствие эффекта Яна-Теллера. Поскольку симметрия таких октаэдров и симметрия одноосных деформаций совпадают, то восприимчивость подсистемы таких октаэдров к одноосным деформациям выше по 
сравнению с восприимчивостью подсистемы подобных, но недеформированных октаэдров. При этом восприимчивость будет тем выше, чем больше степень тетрагональной деформации октаэдра. В манганитах степень тетрагональной деформации октаэдров довольно велика [9], что существенным образом способствует появлению деформационной „мягкости“ кристалла. Однако по мере увеличения ПП $\chi_{3}$, т.е. роста степени упорядочения, „жесткость“ кристалла будет возрастать.

В фазе $\mathrm{O}^{\prime}$ кристалла помимо значительных одноосных деформаций наблюдаются и относительно большие ромбические деформации. Об их росте можно судить по разнице величин отношений $b / a$ в фазах $\mathrm{O}$ и $\mathrm{O}^{\prime}$, где $b$ и $a$ - решеточные постоянные в фазах Pbnm. Так $b / a$ в фазе $\mathrm{O}^{\prime}$ при $523 \mathrm{~K}$ равно $\sim 1.03$, при $653 \mathrm{~K}$ $\sim 1.027$, а в фазе О при $823 \mathrm{~K} \sim 1.002$, при $903 \mathrm{~K}$ $\sim 1.003$, при $973 \mathrm{~K} \sim 1.004$ [15]. Отсюда видно, что ромбические деформации в фазе $\mathrm{O}^{\prime}$ на порядок превышают аналогичные деформации в фазе О. Их рост в фазе $\mathrm{O}^{\prime}$ в основном связан с ростом ПП $\chi_{3}$, поскольку ромбические деформации $\sim \varphi_{1} \varphi_{2}+\psi_{3} \chi_{3}$, но при этом ПП $\varphi$ и $\psi$ возрастают незначительно, а ПП $\chi_{3}$ увеличивается почти на порядок. Необходимо отметить, что в рамках используемого приближения при модельном рассмотрении влияние ПП $\chi_{3}$ на сдвиговые деформации не учитывалось. Таким образом вследствие роста одноосных и ромбических деформаций соотношения между основными параметрами решетки существенно изменятся. Так, если в фазе О имело место соотношение $a<c / \sqrt{2}<b$, то в фазе $\mathrm{O}^{\prime}-c / \sqrt{2}<a<b$. Такое изменение соотношения между структурными параметрами свидетельствует об изменении основного структурного мотива кристалла.

Также необходимо отметить, что фаза $\mathrm{O} \mathrm{LaMnO}_{3}$ оказывается деформированной в большей степени по сравнению с аналогичной фазой $\mathrm{LaFeO}_{3}[8,15]$ - соединения очень близкого по кристаллохимическим параметрам компонент кристаллу $\mathrm{LaMnO}_{3}$. По-видимому, это также связано с деформационной „мягкостью“ фазы О $\left(\mathrm{LaMnO}_{3}\right)$ и наличием в ней малого ПП $\chi_{3}$.

Соотношение между $E$ и $A$ выбрано с учетом того, что $E / A=-\varphi^{2} \psi_{3}$. В $\mathrm{LaMnO}_{3}$ углы поворотов октаэдров в фазе $\mathrm{O} \sim 12-13^{\circ}$ при $T \gtrsim T_{J T}[6,15]$, поэтому $\varphi^{2} \psi_{3} \sim \sin ^{3}\left(12-13^{\circ}\right) \sim 0.01$. Как уже отмечалось, с ростом поля $E$ фазовый переход между изосимметричными фазами становится непрерывным. На качественном уровне изменение рода орбитального ФП можно проиллюстрировать следующим образом. В случае $B=0.82 A$, ФП становится непрерывным при $E \geq-0.03 A$ (рис. 4). Если $E=-0.03 A$, то „переход“ проявится при $T_{\mathrm{tr}} \sim 770 \mathrm{~K}$. При этом углы поворотов октаэдров в фазе О будут $\sim 16-18^{\circ}$.

Подобное изменение параметров орбитального ФП наблюдается в ряду соединений $\mathrm{LaMnO}_{3}, \mathrm{PrMnO}_{3}$, $\mathrm{NdMnO}_{3}$ [9-11] и др., в которых последовательно уменьшается катион $A$. Обычно влияние размеров катиона $A$ на структуру кристалла $\mathrm{ABO}_{3}$ в первом (грубом) приближении оценивают по величине фактора толерантности $t=R_{\mathrm{AO}} / \sqrt{2} R_{\text {Bо }}$, где $R_{\mathrm{AO}}=R_{A}(12)+R_{O}(6)$, $R_{\mathrm{BO}}=R_{B}(6)+R_{O}(6), R_{L}(m)-$ ионный радиус иона $L \mathrm{c}$ координационным числом $m$. Если $t<1$, то связи АО растянуты, а связи ВО - сжаты. Величина $1-t$ характеризует степень растяжения и сжатия связей. В свою очередь растяжение $\mathrm{AO}$ связей и сжатие ВО связей способствует изгибу $\mathrm{B}-\mathrm{O}-\mathrm{B}$ связей за счет поворотов октаэдров $\mathrm{BO}_{6}$.

При уменьшении размера катиона $A$ (уменьшении фактора $t$ ) степень растяжения связей АО растет, увеличивается и „сжатие кристалла“, что приведет к росту изгиба связей $\mathrm{Mn}-\mathrm{O}-\mathrm{Mn}$ и увеличению углов поворота октаэдров $\mathrm{MnO}_{6}$. Поэтому по мере уменьшения катиона $A$ температуры ротационных фазовых переходов увеличиваются, углы поворотов октаэдров и ротационные ПП растут, поле $\sim \varphi^{2} \psi_{3}$ также растет, а когда оно достигнет критического значения орбитальный ФП станет непрерывным. Именно это наблюдается при переходе от $\mathrm{LaMnO}_{3}$ к $\mathrm{PrMnO}_{3}, \mathrm{NdMnO}_{3}$ и др. Так температуры фазовых переходов из кубической в ромбоэдрическую и из ромбоэдрической в фазу О последовательно заметно увеличиваются, углы поворотов октаэдров $\alpha$ также растут, в $\mathrm{LaMnO}_{3}$ угол $\alpha \sim 12-13^{\circ}$, а в $\mathrm{PrMnO}_{3}$ и $\mathrm{NdMnO}_{3} \sim 16-18^{\circ}[9,10]$. Соответственно ротационные ПП и „внешнее поле“ $\sim \varphi^{2} \psi_{3}$ в последних также заметно больше чем в первом соединении. При этом орбитальный ФП в последних становится либо близким к непрерывному, либо непрерывным.

В работе [10] получена зависимость параметров кристаллической решетки $\mathrm{PrMnO}_{3}$ от температуры в области орбитального ФП. Характер нелинейности температурной зависимости постоянных решетки [10] подобен тому, что изображено на рис. 4. Такое сходство дает основание утверждать, что в этом случае имеет место трансформация ФП первого рода в непрерывный под влиянием „внешнего поля“ (рис. 4). Поскольку, как уже отмечалось, реализация ФП первого рода осуществляется при условии „размягчения“ не только по ПП $\chi$, но и по ПП $\eta$, то в данном случае изосимметрийный $\Phi П \mathrm{O} \rightarrow \mathrm{O}^{\prime}$ также происходит между фазами „мягкими“ относительно одноосных деформаций.

\section{5. Заключение}

Наличие „сильно деформирующих“ ЯТ катионов в узлах $B$ решетки перовскита [21] приводит к появлению подсистемы тетрагонально деформированных октаэдров, имеющих в высокотемпературной, симметричной фазе три равноправные ориентации вдоль направлений типа [001] кубической решетки. При соответствующих условиях наличие такой подсистемы будет способствать возникновению деформационной „мягкости“ для одноосных деформаций и появлению ориентационного (орбитального) упорядочения. 
Возникновение ротационно упорядоченной фазы $\mathrm{O}$, характеризуемой ПП $\varphi \varphi \psi(P b n m)$, сопровождается появлением орбитального упорядочения, описываемого ПП $\chi$. В этой фазе основной структурный мотив формируется ротационным упорядочением, а появившийся ПП $\chi$ мал по величине. Тем не менее ПП $\chi$ способен оказывать влияние на структурные и физические свойства соединения. Так наличие этого ПП в фазе О приводит к тому, что „собственно“ орбитальный ФП становится изосимметрийным, а фаза О в манганитах оказывается деформированной в большей степени чем фаза О в ортоферритах.

Фазовый переход типа „порядок-беспорядо“, обусловленный потерей ориентационной устойчивости в подсистеме ЯТ деформированных октаэдров (т.е. „собственно“ орбитальный ФП), является переходом во „внешнем поле“, в качестве которого выступает произведение компонент ротационных ПП $\varphi \varphi \psi$. В зависимости от величины поля (т.е. от величины ротационных ПП) ФП может сопровождаться „скачком“ ПП, характеризующего орбитальное упорядочение, а может быть непрерывным, но сопровождаться сильной нелинейностью в температурной зависимости и ПП и структурных параметров кристалла. При этом, ФП происходит между деформационно неустойчивыми состояниями и сопровождается появлением относительно больших одноосных деформаций, а также заметным увеличением ромбических деформаций за счет роста ПП $\chi$.

Таким образом основные изменения в структуре фазы О' относительно фазы О обусловлены: во-первых появлением собственно орбитального упорядочения в условиях деформационной „мягкости“ структуры; вовторых - совокупным эффектом взаимодействия орбитальных и ротационных упорядочений.

При орбитальном упорядочении заметно меняются расстояния между обменно связанными ионами $\mathrm{Mn}^{3+}$, а также наблюдается уменьшение угла связи $\mathrm{Mn}-\mathrm{O}-\mathrm{Mn}$ по мере уменьшения размера катиона $A$. Именно „укладка“ ЯТ искаженных кислородных октаэдров, отвечающая орбитальному упорядочению, с нашей точки зрения, обусловливает большое отличие параметров решеток орторомбических фаз манганитов и ортоферритов, что приводит к принципиальному отличию их магнитных структур и характеристик фазовых переходов. Для кристаллов ортоферритов характерно антиферромагнитное упорядочение магнитных ионов, входящих в элементарную ячейку, повторяющуюся при трансляциях, которые характеризуют исходную кристаллическую структуру фазы Pbnm. Другими словами, в ортоферритах наблюдается магнитный порядок $G$ типа [8], характеризуемый вектором $\mathbf{k}=0$. В манганитах $\mathrm{AMnO}_{3}$ (A от $\mathrm{Eu}$ до Но) соседние магнитные ионы входящие в разные ячейки, стремятся упорядочиться антиферромагнитно, т.е. ниже $T_{N}\left(T_{N} \gtrsim 50 \mathrm{~K}\right)$ подсистема магнитных катионов теряет устойчивость относительно упорядочения с $\mathbf{k}=(0,1 / 2,0)$ и объем элементарной ячейки увеличивается. Это приводит к тому, что при $T \leq T_{N}$ в этих соединениях возникает несоразмерная магнитная фаза. При дальнейшем понижении температуры в $\mathrm{EuMnO}_{3}$ и $\mathrm{GdMnO}_{3}$ появляется магнитное упорядочение А типа, в соединениях с $\mathrm{A}=\mathrm{Tb}$, Dy происходит ФП в другую несоразмерную фазу, сопровождаемый появлением поляризации, а в $\mathrm{HoMnO}_{3}$ возникает соразмерная магнитная фаза $E$ типа $(\mathbf{k}=(0,1 / 2,0))[4]$.

Соответствующие магнитные ФП описываются параметрами порядка, преобразующимися по двумерным физически неприводимым представлениям векторов $\mathbf{k}$, лежащих на границе зоны Бриллюэна. Эти представления не удовлетворяют условию Лифшица [30] и, следовательно, допускают существование градиентных инвариантов, обусловливающих появление несоразмерных фазовых состояний. Как показано в [31,32], вхождение векторного НП в антисимметричный квадрат параметра порядка (в данном случае $L_{1}, L_{2}$ ), свидетельствующее о существовании инварианта Лифшица $\left(L_{1} \partial L_{2} / \partial x_{i}-L_{2} \partial L_{1} / \partial x_{i}\right)$, означает присутствие данного НП в симметричной части четвертой степени двумерного НП. В рассматриваемом случае соответствующий инвариант имеет вид $L_{1} L_{2}\left(L_{1}^{2}-L_{2}^{2}\right) P_{i}$, где $P_{i}$ - компонента поляризации вдоль направления $x_{i}$. Кроме того, пространственная группа Pbnm, к которой относятся низкотемпературные манганиты, допускает существование взаимодействия вида $L_{1} L_{2} P_{k}\left(P_{k}-\right.$ ортогональная $P_{i}$ компонента вектора поляризации $\left.\mathbf{P}\right)$. Все это справедливо для всех шести НП группы Pbnm, относящихся к векторам обратной решетки вида $(1 / 2,0,0)$. Общим заключением является обязательное присутствие несоразмерных магнитных фаз перед переходом в однородное фазовое состояние и наличие электрической поляризации как в несоразмерных, так и однородных магнитных фазах. Отметим также, что в рассматриваемых манганитах обнаружены и структурные переходы с $\mathbf{k} \neq 0$. Так в [33] показано, что такой переход имеет место в системе $\mathrm{Pr}_{0.6} \mathrm{Ca}_{0.4} \mathrm{MnO}_{3}$. Поэтому в этих соединениях следует ожидать появление структурно несоизмеримых фаз.

\section{Финансирование}

Работа выполнена при финансовой поддержке Министерства науки и высшего образования РФ в рамках базовой части госзадания (грант № 3.5710.2017/8.9).

\section{Конфликт интересов}

Авторы заявляют, что у них нет конфликта интересов.

\section{Список литературы}

[1] Y. Tokura, N. Nagaosa. Science 288, 462 (2000).

[2] J.B. Goodenough. Annu. Rev. Mater. Sci. 28, 1 (1998).

[3] J.M.D. Coey, M. Viret, S. von Molnar. Adv. Phys. 58, 571 (2009).

[4] T. Kimura, S. Ishihara, H. Shintani, T. Arima, K.T. Takahashi, K. Ishizaka, Y. Tokura. Phys. Rev. B 68, 060403(R) (2003). 
[5] J.-S. Zhou, J.B. Goodenough. Phys. Rev. B 68, 144406 (2003).

[6] J. Rodriguez-Carvajal, M. Hennion, F. Moussa, A.H. Moudden, L. Pinsard, A. Revcolevschi. Phys. Rev. B 57, R3189 (1998).

[7] В.Е. Найш. Физика металлов и металловедение 85, 5 (1998).

[8] S.M. Selbach, J.R. Tolchard, A. Fossdal, T. Grande. J. Solid State Chem. 196, 249 (2012).

[9] M.C. Sanchez, G. Subias, J. Garcia, J. Blasco. Phys. Rev. Lett. 90, 045503 (2003).

[10] D. Sanchez, J.A. Alonso, M.J. Martinez-Lope. J. Chem. Soc., Dalton Trans. 4422 (2002).

[11] C.V. Colin, A.J.C. Buurma, M.V. Zimmermann, T.T.M. Palstra. J. Phys.: Condens. Matter 20, 434223 (2008).

[12] A.M. Glazer. Acta Cryst. B 28, 3384 (1972).

[13] K.S. Aleksandrov, J. Bartolome. Phase Transitions, 74, 255 (2001).

[14] В.Е. Найш. Физика металлов и металловедение 92, 3 (2001).

[15] P.M.M. Thygesen, C.A. Young, E.O.R. Beake, F.D. Romero, L.D. Connor, T.E. Proffen, A.E. Phillips, M.G. Tucker, M.A. Hayward, D.A. Keen, A.L. Goodwin. Phys. Rev. B 95, 174107 (2017).

[16] H. Hazama, T. Goto, Y. Nemoto, Y. Tomioka, A. Asamitsu, Y. Tokura. Phys. Rev. B 62, 15012 (2000).

[17] M. Saint-Paul, P. Lejay. Physica B 352, 353 (2004).

[18] X. Qui, Th. Proffen. Phys. Rev. Lett. 94, 177203 (2005).

[19] M.A. Carpenter, C.J. Howard. Acta Cryst. В 65, 134 (2009).

[20] К.И. Кугель, Д.И. Хомский. Успехи физических наук 138, 621 (1982).

[21] J.D. Dunitz, L.E. Orgel. J. Phys. Chem. Solids 3, 20 (1957).

[22] A. Sartbaeva, S.A. Wells, M.E. Thorpe, E.S. Božin, S.J.L. Billinge. Phys. Rev. Lett. 99, 155503 (2007).

[23] Y. Murakami, J.P. Hill, D. Gibbs, M. Blume, I. Koyama, M. Tanaka, H. Kawata, T. Arima, Y. Tokura. Phys. Rev. Lett. 81, 582 (1998).

[24] М.П. Ивлиев, В.П. Сахненко. В сб. тр. Х научно-практического семинара (6-9 июня 2017 г.) Махачкала. С. 8-24.

[25] М.П. Ивлиев, В.П. Сахненко. ФТТ 58, 2340 (2016).

[26] H.T. Stokes, D.M. Hatch. Isotropy subgroup of 230 crystallografic space groups. World-Scientific, Singapore (1988). $573 \mathrm{p}$.

[27] М.П. Ивлиев. ФТТ 51, 1472 (2009).

[28] E.O. Wollan, W.C. Koehler. Phys. Rev. 100, 545 (1955).

[29] M. Kataoka. J. Phys. Soc. Jpn 70, 2353 (2001).

[30] Л.Д. Ландау, Е.М. Лифшиц. Статистическая физика. Наука, М. (1978). Ч. 1. 584 c.

[31] И.Л. Крайзман, В.П. Сахненко. Кристаллография 31, 167 (1986).

[32] С.М. Новиков, В.П. Сахненко. ФТТ 31, 136 (1989).

[33] В.С. Шахматов, Н.М. Плакида, Н.С. Тончев. Письма в ЖЭТФ 77, 18 (2003).

Редактор Т.Н. Василевская 